\title{
Antigenic and genomic homogeneity of successive Mycoplasma hominis isolates
}

\author{
LISE T. JENSEN, P. THORSEN*, B. MøLLER*, S. BIRKELUND and G. CHRISTIANSEN \\ Department of Medical Microbiology and Immunology, Bartholin Building, University of Aarhus, DK-8000 \\ Aarhus C and *Department of Obstetrics and Gynaecology, Odense University Hospital, DK-5000 Odense C, \\ Denmark
}

\begin{abstract}
Sixty Mycoplasma hominis isolates were obtained from the cervices of pregnant women and from the ears or pharynges of their newborn babies. The isolates were examined by SDS-PAGE and pulsed-field gel electrophoresis. Antigenic and genomic profiles were obtained for 16 series with two or more successive isolates. Both analyses led to the conclusion that isolates from the same woman were identical or nearly identical, while isolates from different women exhibited a high degree of variation with respect to both genomic and antigenic profiles.
\end{abstract}

\section{Introduction}

Mycoplasma hominis is one of $>70$ species comprising the class Mollicutes with a characteristically small genome size, an absence of cell wall and a generally low $\mathrm{G}+\mathrm{C}$ content. Despite the limited coding capacity ranging from $580 \mathrm{~kb}$ ( $M$. genitalium [1]) to $1300 \mathrm{~kb}$ ( $M$. iowae [2]), mycoplasmas have adapted to a wide variety of hosts and are found to colonise mainly mucous tissues in man and animals. The lack of a cell wall exposes the plasma membrane directly to the environment, making the ability to alter surface proteins of critical importance for colonisation and survival of the micro-organism.

$M$. hominis is a sexually transmitted human pathogen and a frequent inhabitant of the genital tract. Despite years of intensive studies, the role of $M$. hominis in disease is not yet clear. However, there is an association with a number of urogenital diseases $[3,4]$. Extragenital infections, particularly in immunosuppressed individuals, are being reported with increasing frequency $[5,6]$. Assessment of the pathogenic potential of $M$. hominis is complicated by a high degree of genomic and antigenic heterogeneity within the species. The picture is further complicated by the fact that the micro-organism is isolated frequently from asymptomatic males and females [7].

Received 31 July 1997; accepted 10 Nov. 1997

Corresponding author: Dr G. Christiansen.
Variability among different strains of $M$. hominis has been studied by several approaches. Ladefoged and Christiansen [8] constructed physical and genetic maps of the genomes of five $M$. hominis strains obtained from different individuals. Genome sizes obtained by pulsed-field gel electrophoresis ranged from 704 to $825 \mathrm{~kb}$. Restriction patterns varied greatly; none of the strains had identical restriction patterns with any of the five restriction endonucleases used. However, the order of the mapped genes was conserved in the strains. The five strains were included previously in a study by Andersen et al. [9]. They compared the antigenic profiles of $14 \mathrm{M}$. hominis strains by SDSPAGE, two-dimensional (2-D) gel electrophoresis and immunoblotting. It was possible to distinguish each of the 14 strains despite high similarities (76-99\%) between the strains in SDS-PAGE. The electrophoretic 2-D protein patterns had lower similarities ranging from 41 to $72 \%$ between individual isolates. As 2-D gel electrophoresis separates proteins according to both mol.wt and iso-electric point, these lower similarity data seem to be more accurate than those obtained by SDS-PAGE. No two isolates were found to be identical by either of the two above-mentioned methods or by the immunoblotting analyses. Christiansen and Andersen [10] studied genetic variation of $26 M$. hominis strains as reflected in differences in hybridisation patterns. DNA from the 26 strains was probed by Southern blotting with plasmids containing parts of the relatively conserved mycoplasma ribosomal RNA (rRNA) genes. Variability in restriction patterns was frequent outside the rRNA operons and was observed within the rRNA genes in three strains. The 26 strains were classified on the basis of RFLP 
patterns. Three classes contained six, three and two strains, respectively, with identical RFLP patterns. A fourth class contained the remaining 15 strains with unique restriction cleavage patterns. The finding that 15 of the 26 strains showed unique patterns was surprising, as genes encoding rRNA are among the most conserved genes in prokaryotic cells. In mycoplasmas, which harbour only one or two rRNA gene copies, it seems crucial to maintain functional rRNA gene operons. Therefore, the heterogeneity observed within these genes probably reflects a 'minimum of variation' detectable in $M$. hominis. The apparently identical strains with respect to RFLP patterns in rRNA genes were not the most related strains with respect to protein profiles as determined in the study by Andersen et al. [9].

The very heterogeneous character of $M$. hominis combined with a possibly minimal coding capacity, the lack of cell wall and the occasionally opportunist behaviour make the organism an interesting subject in studies of not only $M$. hominis pathogenesis, but also with respect to the general development of bacterial mechanisms of surface variation.

This study reports the variability among $60 \mathrm{M}$. hominis isolates randomly chosen from a recent study by Thorsen [11]. He evaluated the association between various micro-organisms, including $M$. hominis, isolated from the cervices of 3178 pregnant women, and bacterial vaginosis. Among the 60 isolates, 16 series of two or more isolates from the same woman or her newborn baby, or both, were examined, and the variability among these successive isolates was compared with the variability among isolates from different women. Protein profiles were analysed by SDS-PAGE and genomic profiles were evaluated by pulsed-field gel electrophoresis (PFGE) of genomic DNA cleaved with two different restriction endonucleases.

\section{Materials and methods}

\section{Mycoplasma strains and cultivation}

Sixty $M$. hominis isolates were obtained from pregnant women and their newborn babies participating in a cross-sectional population-based study [11]. The numbers refer to the woman and the letters B, G, S, M and $\mathrm{N}$ refer to the relative time of recovery: $\mathrm{B}, \mathrm{G}$ and $\mathrm{S}$, indicating the first, second and third antenatal hospital visits, respectively; and $M$ and $N$ indicating samples taken from the ear and pharynx, respectively, of the newborn baby. Samples to be analysed for the presence of $M$. hominis were taken with sterile cotton-tipped swabs and inoculated directly into $1 \mathrm{ml}$ of $\mathrm{BE}$ liquid growth medium [12], and frozen at $-80^{\circ} \mathrm{C}$ for later cultivation. Cultures were grown in BEA medium [12] and filter cloned once according to the recommendations of the Subcommittee of the Taxonomy of Mollicutes [13].

\section{SDS-PAGE and immunoblotting}

Whole-cell preparations of $M$. hominis harvested in log-phase were dissolved in sample buffer containing $62.5 \mathrm{mM}$ Tris $\mathrm{HCl}(\mathrm{pH} \mathrm{6.8)}$, glycerol $10 \% \mathrm{v} / \mathrm{v}$, SDS $2.3 \% \mathrm{w} / \mathrm{v}, \beta$-mercaptoethanol $5 \% \mathrm{v} / \mathrm{v}$ and bromophenol blue $0.05 \% \mathrm{w} / \mathrm{v}$. The samples were boiled for $5 \mathrm{~min}$ and subjected to electrophoresis in polyacrylamide $7.5 \%$ gels followed by Coomassie Blue staining as described previously [9].

\section{Construction of dendrogram}

Coomassie Blue-stained SDS-PAGE gels were analysed for the presence or absence of 35 distinct protein bands. A dendrogram based on an average linkage algorithm was constructed from these data by computer programmes (ETDIV and ETCLUS, v.2.3), kindly provided by T. S. Whittam (Institute of Molecular Evolutionary Genetics, University of Pennsylvania, USA).

\section{Preparation, restriction endonuclease digestion and PFGE of DNA in agarose blocks}

Six $\mathrm{ml}$ of $M$. hominis cultures in log-phase were harvested by centrifugation at $15000 \mathrm{rpm}$ for $40 \mathrm{~min}$ and washed twice with phosphate-buffered saline (PBS; $20 \mathrm{mM}$ sodium phosphate, $250 \mathrm{mM} \mathrm{NaCl}, \mathrm{pH} 7.4$ ). The pellets were resuspended in $150 \mu \mathrm{l}$ of PBS, mixed with $150 \mu \mathrm{l}$ of low-melting-point agarose (Incert agarose; FMC Bioproducts, Rockland, MN, USA) $1.8 \%$ in PBS, previously melted and equilibrated to $43^{\circ} \mathrm{C}$. The suspension was immediately transferred to a plastic mould $(20 \times 9 \times 1.2 \mathrm{~mm})($ BioRad Laboratories, Richmond, CA, USA) which had been cooled on ice to ensure instant setting of the gel. The agarose plugs were transferred to tubes containing lysis buffer (sarcosyl 1\%, 0.5 M EDTA, $10 \mathrm{mM}$ Tris- $\mathrm{HCl}, \mathrm{pH} 9.5$ ) with Proteinase $\mathrm{K}$ (Boehringer $\mathrm{GmbH}$, Mannheim, Germany) $200 \mathrm{mg} / \mathrm{L}$. After incubation for $2 \mathrm{~h}$ at $50^{\circ} \mathrm{C}$, the lysis buffer was changed and the plugs were incubated overnight at $50^{\circ} \mathrm{C}$. The plugs were washed with lysis buffer without proteinase $\mathrm{K}$ for $2 \mathrm{~h}$ and stored in $100 \mathrm{mM}$ EDTA. Before endonuclease digestion, small blocks $(3 \times 4.5 \times 1.2 \mathrm{~mm})$ were washed for $1 \mathrm{~h}$ with several changes of TE buffer $(10 \mathrm{mM}$ Tris $\mathrm{HCl}, 1 \mathrm{~mm}$ EDTA, $\mathrm{pH} \mathrm{8.0)}$, and equilibrated in $200 \mu \mathrm{l}$ of the appropriate restriction endonuclease buffer at room temperature. Digestion was carried out for $2 \mathrm{~h}$ in $120 \mu \mathrm{l}$ restriction endonuclease buffer, 20 units of enzyme and bovine serum albumin (Boehringer Mannheim) $12 \mu \mathrm{g}$ at temperatures specified by the supplier.

Before electrophoresis the blocks were washed in TE buffer for $30 \mathrm{~min}$, followed by equilibration in $0.5 \times$ TBE buffer $(44.5 \mathrm{mM}$ Tris-borate, $44.5 \mathrm{mM}$ boric acid, $1 \mathrm{mM}$ EDTA, pH 8.0). Agarose 1\% gels in $0.5 \times \mathrm{TBE}$ buffer were run for $20 \mathrm{~h}$ in $0.5 \times \mathrm{TBE}$ buffer at $14^{\circ} \mathrm{C}$ and $200 \mathrm{~V}$ in the CHEF-DR II system (BioRad) and the sub-cell electrophoresis cell (Bio- 
Rad), respectively. Pulse times were either $1-15$ s or $2-$ $23 \mathrm{~s}$. DNA bands were visualised by ethidium bromide staining after electrophoresis. A lambda DNA ladder (Biolabs, New England, USA) and Hind III-digested lambda DNA were used as mol. wt standards.

\section{Results}

\section{SDS-PAGE and antigenic cluster analysis}

For each isolate $30-40$ protein bands were observed, of which c. 20 were consistently present (Fig. 1). The variability in banding pattern was most pronounced for the high mol.wt bands, while smaller proteins were more consistently observed. Despite the often pronounced similarities, a marked heterogeneity between isolates from different women was evident. Isolates originating from the same woman were identical or differed only by the presence of one or two protein bands (Fig. 1).

To examine whether the isolates could be grouped in antigenically related clusters, a dendrogram was constructed on the basis of protein profiles obtained by SDS-PAGE. Coomassie Blue-stained gels were analysed for the presence or absence of 35 distinct protein bands (indicated at the top, Fig. 1). The quantity of individual polypeptides was not taken into account. The data were processed by the computer programmes ETDIV and ETCLUS and a dendrogram of relatedness was constructed (Fig. 2). The isolates were evenly distributed and no clustering could be observed. Isolates originating from the same woman were identical or closely localised on the dendrogram, reflecting a high degree of homogeneity. The five isolates from series 1720 had identical protein profiles as did the series 1697, 2462, 2641 and 2509. With three isolates in series 2867 , the first (B) and the second (G) differed by a single protein, while the isolate from the newborn baby $(\mathrm{N})$ was identical to the second isolate from the mother. Isolate $1572 \mathrm{~B}$ differed from the two identical isolates from the newborn baby ( $\mathrm{M}$ and $\mathrm{N}$ ) by a single band. Isolate

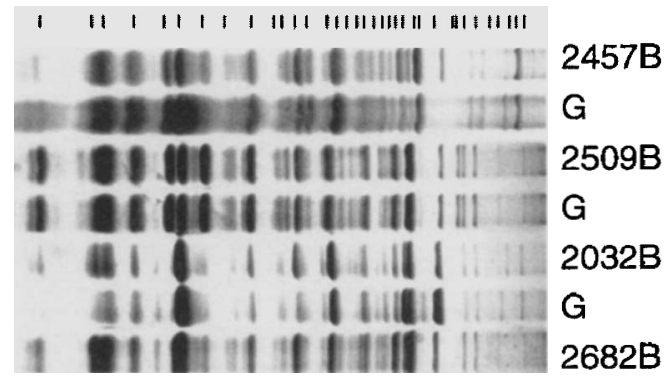

Fig. 1. Coomassie Blue-stained SDS-PAGE gel. Numbers refer to persons and letters refer to the relative time of recovery; $B$, first antenatal hospital visit; $G$, second visit. The locations of 35 distinct protein bands are indicated at the top.
$1762 \mathrm{~B}$ was identical to one of the isolates from the baby $(1762 \mathrm{M})$, but differed by three bands from the other isolate $(1762 \mathrm{~N})$. Several series with two isolates differed by a single mismatch (3052, 2763, 2457 and 2032), two mismatches (2347 and 2682) or three mismatches (2223 and 1935) in protein profiles. Isolates from different women were more distant in the dendrogram; however, some isolates differed by only three mismatches (305B and 327, 519B and the series 2641, 549B and the series 1572). Randomised data input resulted in dendrograms in which identical isolates or isolates with one or two mismatches were always closely localised. Whenever more than two mismatches occurred the localisation in the dendrogram must be considered random.

\section{Genomic analysis of isolates}

All isolates were digested with the restriction endonucleases Xho I and SmaI. Restriction patterns of the series and some individual isolates digested with Sma I are shown in Fig. 3a and b, respectively. Genome sizes calculated on the basis of the band patterns varied from 650 to $800 \mathrm{~kb}$. No isolates from different women were identical in both digestions (Fig. 3a and b), in contrast to isolates originating from the same woman. Ten series $(1720,2867,1697,1762,2457,2462,2509,2641,2682$ and 3052) contained identical isolates in both digestions (Fig. 3a). Two series (2223 and 1935) showed variations in both the Xho I and Sma I digestions (Figs. $3 \mathrm{a}$ and 4). In addition to the bands seen in 2223B, $2223 \mathrm{~S}$ exhibited additional bands corresponding to an increased size of $300 \mathrm{~kb}$. As these additional bands were of lower intensity, $2223 \mathrm{~S}$ was considered to be a mixed culture (Fig. 3a). The isolates from series 1935 differed markedly in the intensity of one band $(175 \mathrm{~kb})$ and the presence of another $(40 \mathrm{~kb})$ in the Sma I digestion consistent with a deletion of $>200 \mathrm{~kb}$ in 1935S. Digestion with Xho I showed replacement of bands of 325 and $275 \mathrm{~kb}$ with a band of $300 \mathrm{~kb}$ in 1935 S compared with 1935B (Fig. 4a). However, the $300-\mathrm{kb}$ band could be a double band resulting from comigration of similarly sized fragments. If not, an apparent deletion of $>200 \mathrm{~kb}$ would result in a genome size of $550 \mathrm{~kb}$, which is even smaller than that of $M$. genitalium which possesses the smallest genome $(580 \mathrm{~kb})$ reported so far. Therefore, the endonucleases Apa I, Sal I and Bam HI were used for further analysis of the two isolates (Fig. 4a). While Apa I did not cut either of the genomes, Sal I digestion showed variations of band sizes yet adding up to approximately identical genome sizes. Bam $\mathrm{HI}$ showed variation of two bands. Considering the $35-\mathrm{kb}$ band of $1935 \mathrm{~B}$ as a double band, the genome sizes were thus identical, and the major deletion indicated by the SmaI digestion could be excluded by these findings. The isolates in series 1935 demonstrate the difficulty of interpreting intricate band patterns, mainly caused by co-migration of similar sized fragments and, furthermore, emphasise the need to use different endonucleases for comparison of 


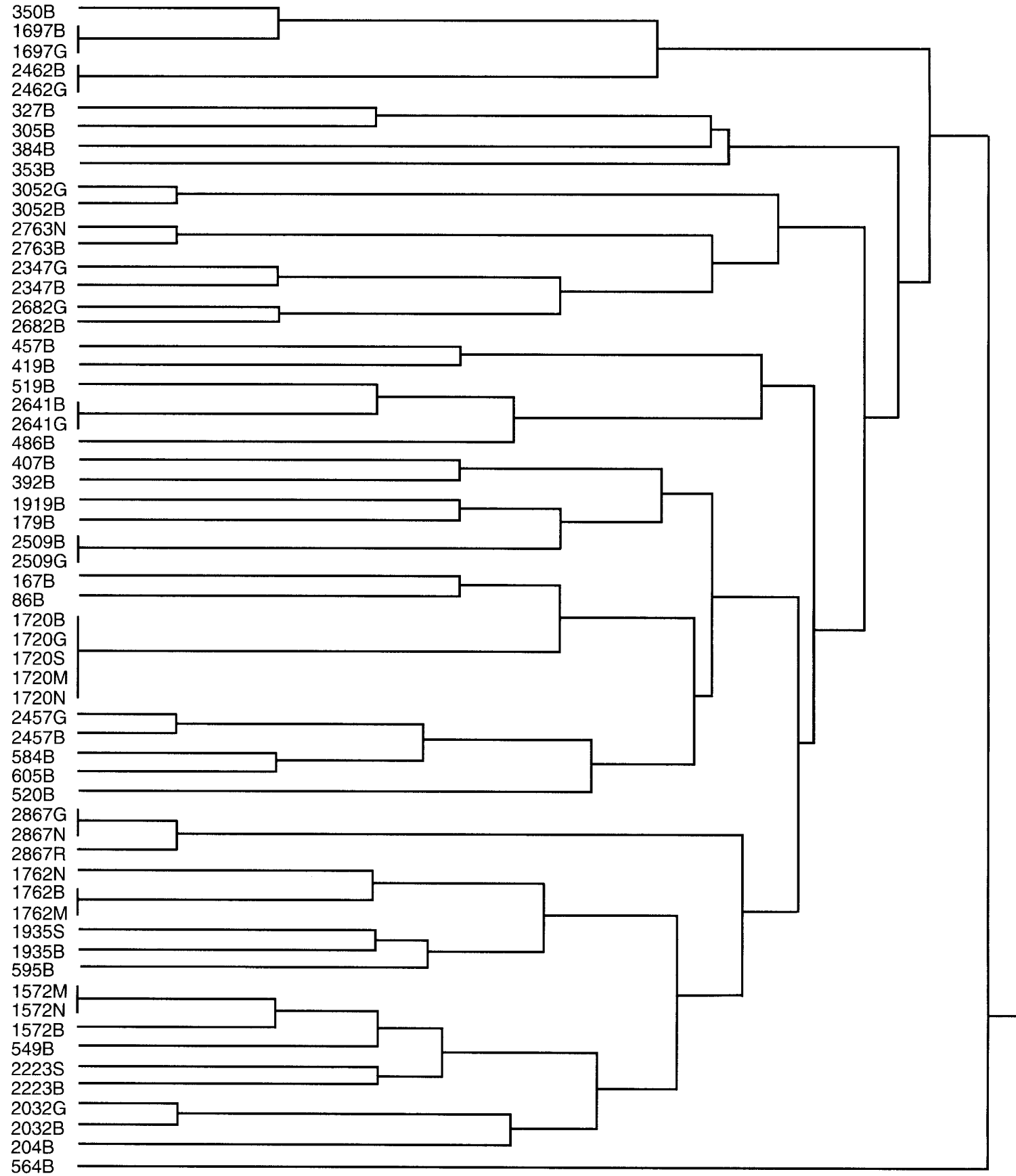

Fig. 2. Dendrogram constructed by the programs ETDIV and ETCLUS (v. 2.3) based on an average linkage algorithm. The distance is measured as the proportion of dissimilarities in the presence or absence of 35 distinct protein bands as determined by SDS-PAGE. Numbers refer to persons and letters to the relative time of recovery; $B$ indicates the first antenatal hospital visit, $\mathrm{G}$ and $\mathrm{S}$, the second and third visits; $\mathrm{M}$ and $\mathrm{N}$ indicate samples taken from the ear and pharynx, respectively, of the newborn baby.

isolates. Four series $(1572,2763,2032,2347)$ showed small variations in only one of the digestions. Isolate 1572B from the mother was identical to isolate $1572 \mathrm{M}$ from the pharynx of the newborn baby. Isolate $1572 \mathrm{~N}$ from the ear of the newborn baby, differed in the Sma I digestion from these two isolates in the sizes of two DNA fragments because of an insertion of $25 \mathrm{~kb}$ (Fig. 3a). The insertion was probably not recognisable in the Xho I digestion because of the relatively small size. Series 2763 showed band patterns indicating a deletion. Compared to the band pattern of the maternal isolate $2763 \mathrm{~B}$, the baby isolate $2763 \mathrm{~N}$ lacked a $125-\mathrm{kb}$ fragment and had an additional $75-\mathrm{kb}$ fragment in the Sma I digestion (Fig. 3a). Neither of the isolates had an Xho I restriction site. Series 2032 gave identical isolates in the Sma I digestion. In the Xho I digestion an additional DNA fragment of $125 \mathrm{~kb}$ was seen in 2032B (Fig. 4b). The band was of low intensity and could have been due a small subpopulation of mycoplasmas exhibiting a frequently found Xho I restriction pattern. It should be kept in mind that the cultures were subcloned only once before use. Series 2347 appeared 
a
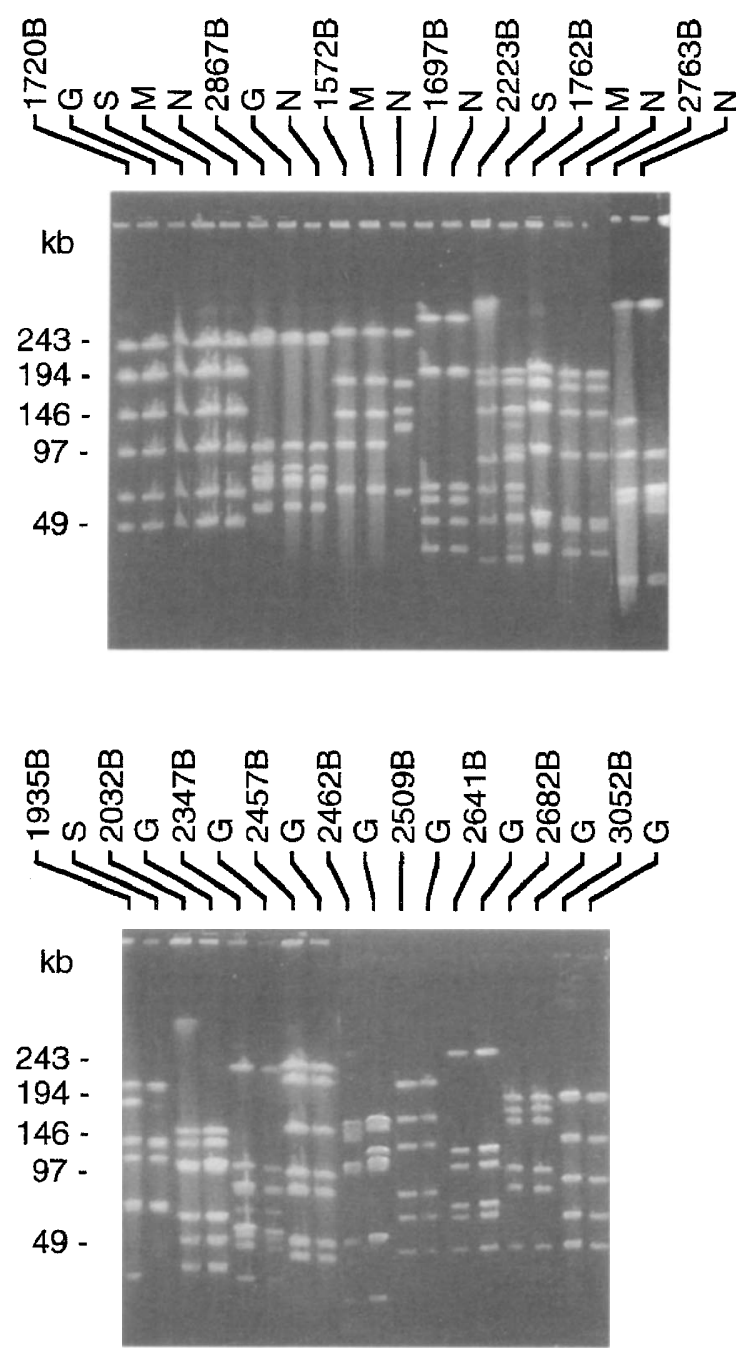

b

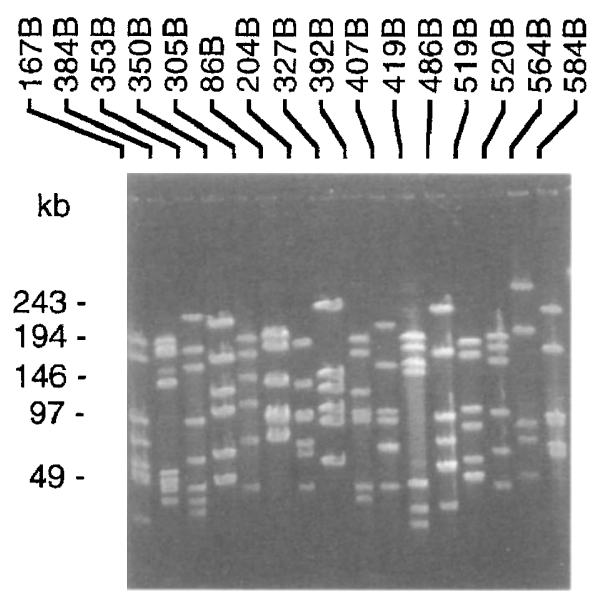

Fig. 3. Ethidium bromide-stained PFGE gels of Sma Icleaved chromosomal DNA of: a, series isolates; $\mathbf{b}$, individual isolates.

to contain identical isolates in the Sma I digestion. A 150-kb deletion, including an Xho I restriction site, could account for the absence of one band seen in the Xho I digestion in isolate $2347 \mathrm{G}$ (Fig. 4c). In the Sma I digestion the band sizes were identical; however, in isolate $2347 \mathrm{~B}$, high intensity staining indicated double bands of 90 and $60 \mathrm{~kb}$ which confirmed the finding of a deletion.

Sma I restriction sites were found in all isolates, whereas four isolates were resistant to Xho I digestion, possibly because of modification/methylation of the recognition site.

Similar band patterns were produced in several isolates when digested with Xho I (data not shown). However, when these isolates were electrophoresed under different conditions, small size differences were seen. The variability was confirmed since different band patterns were observed when the restriction endonuclease Sma I was used. This indicates that the Xho I restriction sites, in contrast to the Sma I restriction sites, are located in relatively conserved gene regions, and emphasises the importance of using more than one restriction endonuclease for comparison of isolates.

\section{Comparison of antigenic and genomic profiles}

Genomic differences were found in series 1572, 2223, 1935, 2032, 2347 and 2763 which could have resulted from mixed cultures. These series also showed differences in antigenic profiles. The major deletion found in isolate $2347 \mathrm{G}$ was antigenically reflected in the absence of two protein bands in SDS-PAGE. However, none of the deletions (series 2347 and 2763) affected the growth of the isolates. Differences in antigenic profiles were further seen in serics 3052 , 2682, 2457, 2867 and 1762. The remaining five series - 1697, 2462, 2641, 2509 and 1720 - showed identity in both genomic and antigenic profiles. While more than half of the series exhibited antigenic or genomic variations, or both, these were small compared with the overall variability as reflected among the series and the individual isolates.

\section{Discussion}

This study investigated the genomic and antigenic variation of $60 \mathrm{M}$. hominis isolates, comprising 16 series of successive isolates. The isolates did not form antigenically related clusters on the basis of SDS-PAGE when analysed with the computer programs ETDIV and ETCLUS. Isolates from the same woman were identical or very similar, whereas isolates from different women exhibited marked antigenic heterogeneity. This finding was supported by the PFGE analysis, in which a substantial genomic variation was revealed within isolates originating from different women. In contrast, isolates originating from the same woman had identical genomic profiles in 10 of the 16 series. The isolates from series 2032 and 2223 exhibited differences. As the intensity and presence of bands varied between the 
a

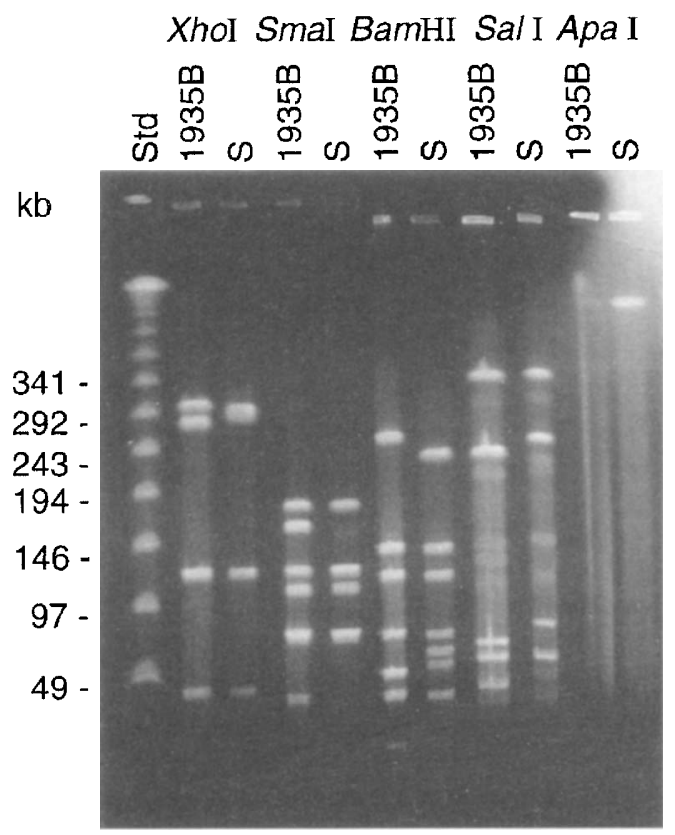

b

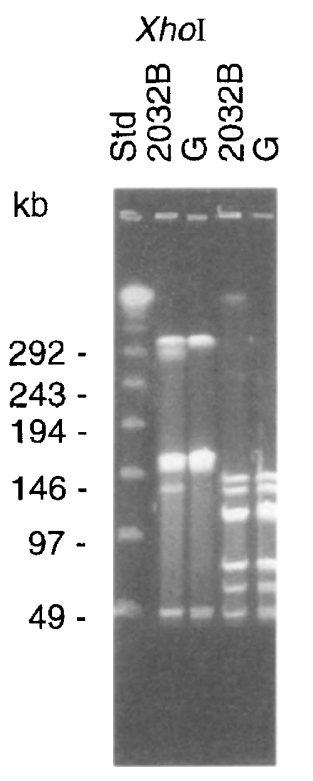

C

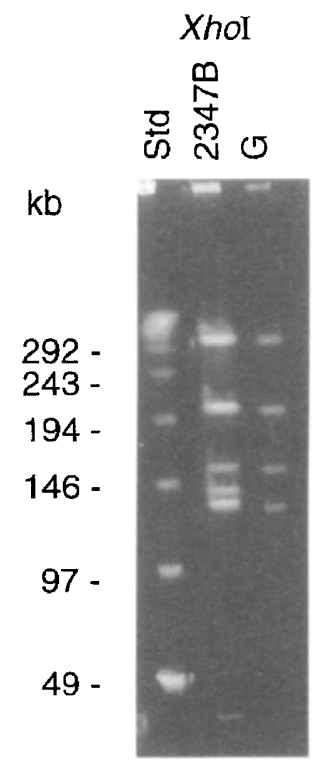

Fig. 4. Ethidium bromide-stained PFGE gels of series isolates differing in at least one endonuclease digestion: a, series isolates $1935 \mathrm{~B}$ and $1935 \mathrm{~S}$ cleaved with five different restriction endonucleases; b, series isolates 2032B and 2032G cleaved with Xho I and Sma I; c, series isolates $2347 \mathrm{~B}$ and $2347 \mathrm{G}$ cleaved with Xho I. The mol. wt standard is shown on the left.

isolates, and as the isolates were filter-cloned only once before use, these differences were considered to result from mixed cultures. Differences between the isolates from series 2763 and 2347 probably resulted from genomic deletions, while an insertion most probably accounted for the differences in band pattern observed for series 1572. Series 1935 showed intricate band patterns when digested with five different enzymes, probably due to several double bands. Despite varying band patterns, genome sizes in four digestions were identical, suggesting that substitutions at cleavage sites and inversions accounted for the observed variations. The differences for some series were seen in only one of the digestions. This may be because the relatively small differences of fragment sizes could not be distinguished in the high mol. wt bands.

Studies of variability have previously been performed on isolates of miscellaneous origin [14-16]. However, the use of consecutive isolates to examine antigenic variation might provide valuable information on possible mechanisms used by the pathogen to change antigenically over time. Only a single report has described antigenic variation in successive $M$. hominis isolates [17]. Olson et al. examined the expression of surface antigens of 14 consecutive isolates obtained from the synovial fluid of a patient with chronic septic arthritis. Monoclonal antibodies (MAbs) were raised against the primary $M$. hominis isolate and subsequently used in immunoblotting with all isolates. The isolates differed markedly in protein profiles, while restriction and hybridisation patterns were virtually identical when fragments of the conserved rRNA operon and elongation factor $\mathrm{Tu}$ gene were used as probes. When these probes were used in hybridisation studies with isolates obtained from different sources, a high degree of variation in hybridisation patterns was obtained. This supports the assumption that, in the study by Olson et al. [17], antigenic variation rather than re-infection had occurred.

A high degree of heterogeneity as demonstrated for several mycoplasma species reflects a rapid evolution. This could be explained by either a general genomic instability or by evolution caused by selective pressure. The results of the present study indicate the latter, as isolates from different women exhibited substantial variation, whereas isolates originating from the same woman were found to be nearly identical, suggesting adaptation to the host environment. This confirms a previous study involving characterisation of three antigenic variants of $M$. hominis PG21 selected by culture with MAb 552 added to the growth medium [18]. To establish whether addition of a $\mathrm{MAb}$ to the growth medium could mediate major changes in DNA or protein profiles, the type strain $M$. hominis PG21 was cultured for a 3-month period in the presence of MAb 552, which reacts with epitopes encoded by 471-bp long tandemly repeated sequences identified in the PG21 $\operatorname{lmp}$ genes $[19,20]$. Southern and Western blot analyses were performed on three occasions during culture. The cultures with MAb 552 added to the medium showed deletions of a number of the 471-bp repeats. The genetic deletions were 
accompanied by a decrease in size of the proteins. Control cultures without antibody added to the medium showed no alterations. It was concluded that the observed antigenic variation resulted from in-vitro antibody-mediated selection.

Variation among species is generally studied with respect to either antigenic profiles with MAbs, SDSPAGE and 2-D gel electrophoresis [9, 21-25] or genomic and genetic profiles by DNA-DNA hybridisations, PCR and restriction endonuclease cleavage analysis $[14,16,26]$. In the present study the examination of both antigenic and genomic variability resulted in a limited correlation between genomic RFLP and antigenic properties. Some antigenic differences were not reflected in the PFGE analysis as illustrated by series 2867 and 2682, in which the isolates exhibited identical PFGE patterns. On the other hand, series that showed different PFGE profiles always exhibited minor antigenic differences as reflected in SDS-PAGE. Antigenic variations could result from a number of genomic changes not detectable in PFGE, such as minor insertions or deletions, point mutations, on-off phase shifts and recombination events, or to erroneous transcription or translation. Alternatively, the variation found in the PFGE analysis may only reflect either major chromosomal re-arrangements such as large deletions or insertions, or point mutations specific to the endonuclease restriction sites. Therefore, not surprisingly, the correlation between antigenic and genomic variability was limited to the detection of a general heterogeneity among isolates from different women and homogeneity among isolates from the same women in the present study. Lack of correlation between genomic and antigenic variability was previously reported by Poumarat et al. [27] who examined genomic and antigenic variability of $37 \mathrm{M}$. bovis strains. The isolates were classified into 13 different genomic groups based on restriction endonuclease analysis with three enzymes. Antigenic profiles were investigated by immunoblotting with a polyclonal calf serum and three MAbs. An equally pronounced antigenic variability was observed whether the isolates came from the same or different genomic groups.

Variability in relation to time, geography and anatomical site of isolation is poorly understood. Frey et al. [28] found that the chromosomal heterogeneity as demonstrated by PFGE of $M$. hyopneumoniae strains from different countries was markedly larger than between strains isolated from the same laboratory. Solsona et al. [29] also found that the antigenic variability of $31 \mathrm{M}$. agalactiae strains was related to the geographic origin of the strains. In a study by Barile et al. [30] strains isolated from the same sources were found to form clusters of strains having very high genomic homologies. However, these observations could not be confirmed when antigenic variation among $14 \mathrm{M}$. hominis isolates from different sources and 12 genital isolates was compared. The genital isolates were found to vary as much as the 14 isolates from different sources [31].

The isolates used in the present study were collected within a rather small geographical area and over a period of 18 months. All samples originated from the cervices of pregnant women or from newborn babies. A high degree of heterogeneity was found with respect to both antigenic and genomic variation despite the uniformity in time, geography and anatomical origin of the isolates. Successive isolates in general remained genomically stable over the sampling period, suggesting that the colonisation site and local host immune response might be of considerable importance for the micro-organism to accomplish antigenic variation.

This work was supported by the Danish Health Research Council (grant 12-0850-1 and grant 12-1620-1), Aarhus University Foundation, Novo's Foundation and Fonden til Lœevidenskabens Fremme. We thank Karin S. Sørensen and Inger Andersen for excellent technical assistance.

\section{References}

1. Su CJ, Baseman JB. Genome size of Mycoplasma genitalium $J$ Bacteriol 1990; 172: 4705-4707

2. Grau O, Laigret F, Carle P, Tully JG, Rose DL, Bove JM. Identification of a plant-derived mollicute as a strain of an avian pathogen, Mycoplasma iowae, and its implications for mollicute taxonomy. Int $J$ Syst Bacteriol 1991; 41: 473-478.

3. Mårdh PA. Mycoplasma hominis: a neglected human pathogen. Eur J Clin Microbiol 1983; 2: 303-308.

4. Taylor-Robinson D, McCormack WM. The genital mycoplasmas. $N$ Engl J Med 1980; 302: 1063-1067.

5. Madoff S, Hooper DC. Nongenitourinary infections caused by Mycoplasma hominis in adults. Rev Infect Dis 1988; 10: $602-613$

6. McMahon DK, Dummer JS, Pasculle AW, Cassell GH. Extragenital Mycoplasma hominis infections in adults. Am Med 1990; 89: 275-281

7. Furr PM, Taylor-Robinson D. Prevalence and significance of Mycoplasma hominis and Ureaplasma urealyticum in the urines of a non-venereal disease population. Epidemiol Infect 1987; 98: 353 .

8. Ladefoged SA, Christiansen G. Physical and genetic mapping of the genomes of five Mycoplasma hominis strains by pulsedfield gel electrophoresis. J Bacteriol 1992; 174: 2199--2207.

9. Andersen H, Birkelund S, Christiansen G, Freundt EA Electrophoretic analysis of proteins from Mycoplasma hominis strains detected by SDS-PAGE, two-dimensional gel electrophoresis and immunoblotting. I Gen Microbiol 1987; 133 $181-191$

10. Christiansen G, Andersen H. Heterogeneity among Mycoplasma hominis strains as detected by probes containing parts of ribosomal ribonucleic acid genes. Int J Syst Bacteriol 1988; 38: $108-115$

11. Thorsen P. Bacterial vaginosis in pregnancy. A populationbased study. PhD thesis, Odense University, Denmark. 1997.

12. Freundt EA, Ernø H, Lemcke RM. Identification of mycoplasmas. Methods Microbiol 1979; 13: 377-434.

13. International Committee on Systematic Bacteriology, Subcom mittee on the Taxonomy of Mollicutes. Proposal of minimal standards for descriptions of new species of the class Mollicutes. Int J Syst Bacteriol 1979; 29: 172-180.

14. Blanchard A, Yanez A, Dybvig K, Watson HL, Griffiths G, Cassell $\mathrm{GH}$. Evaluation of intraspecies genetic variation within the 16S rRNA gene of Mycoplasma hominis and detection by polymerase chain reaction. J Clin Microbiol 1993; 31 $1358-1361$.

15. Christiansen G, Ladefoged S, Hauge S, Birkelund S, Andersen $\mathrm{H}$. Use of monoclonal antibodies for detection of gene and 
antigen variation in Mycoplasma hominis. Int $J$ Med Microbiol Suppl 1990; 20: 535-545.

16. Nyvold C, Birkelund S, Christiansen G. The Mycoplasma hominis P120 membrane protein contains a 216 amino acid hypervariable domain that is recognized by the human humoral immune response. Microbiology 1997; 143: 675-688.

17. Olson LD, Renshaw CA, Shane SW, Barile MF. Successive synovial Mycoplasma hominis isolates exhibit apparent antigenic variation. Infect Immun 1991; 59: 3327-3329.

18. Jensen LT, Ladefoged S, Birkelund S, Christiansen G. Selection of Mycoplasma hominis PG21 deletion mutants by cultivation in the presence of monoclonal antibody 552. Infect Immun 1995; 63: 3336-3347.

19. Ladefoged SA, Birkelund S, Hauge S, Brock B, Jensen LT, Christiansen G. A 135-kilodalton surface antigen of $\mathrm{MycO}$ plasma hominis PG21 contains multiple directly repeated sequences. Infect Immun 1995; 63: 212-223.

20. Ladefoged SA, Jensen LT, Brock B, Birkelund S, Christiansen G. Analysis of 0.5-kilobase-pair repeats in the Mycoplasma hominis lmp gene system and identification of gene products. $J$ Bacteriol 1996; 178: 2775-2784.

21. Ladefoged SA, Hauge S, Andersen H, Birkelund S, Christiansen G. Use of monoclonal antibodies for detection of antigen variation in Mycoplasma hominis. Int $J$ Med Microbiol Suppl 1990; 20: 634-639.

22. Olson LD, Shane SW, Karpas AA, Cunningham TM, Probst PS, Barile MF. Monoclonal antibodies to surface antigens of a pathogenic Mycoplasma hominis strain. Infect Immun 1991; 59: $1683-1689$.

23. Panangala VS, Morsy MA, Gresham MM, Toivio-Kinnucan M.
Antigenic variation of Mycoplasma gallisepticum, as detected by use of monoclonal antibodies. Am $J$ Vet Res 1992; 53: $1139-1144$

24. Panangala VS, Gresham MM, Morsy MA. Antigenic heterogeneity in Mycoplasma iowae demonstrated with monoclonal antibodies. Avian Dis 1992; 36: 108-113.

25. Avakian AP, Ley DH, Kleven SH. Comparison of Mycoplasma synoviae isolates by immunoblotting. Avian Pathology 1992; 21: $633-642$.

26. Christiansen G, Andersen H, Birkelund S, Freundt EA. Genomic and gene variation in Mycoplasma hominis strains. Isr J Med Sci 1987; 23: 595-602.

27. Poumarat F, Solsona M, Boldini M. Genomic, protein and antigenic variability of Mycoplasma bovis. Vet Microbiol 1994; 40: $305-321$.

28. Frey J, Haldimann A, Nicolet J. Chromosomal heterogeneity of various Mycoplasma hyopneumoniae Field strains. Int J Syst Bacteriol 1992; 42: 275-280.

29. Solsona M, Lambert M, Poumarat F. Genomic, protein homogeneity and antigenic variability of Mycoplasma agalactiae. Vet Microbiol 1996; 50: 45-58.

30. Barile MF, Grabowski MW, Stephens EB et al. Mycoplasma hominis - Tissue cell interactions: a review with new observations on phenotypic and genotypic properties. Sex Transm Dis 1983; 10(4 Suppl): 345-354

31. Christiansen G. Genetic variation in natural populations. In Maniloff J, McElhaney, Finch RH, Baseman JB (eds) Mycoplasmas: molecular biology and pathogenesis. Washington, DC, American Society for Microbiology. 1992: $561-573$. 